\title{
Shaded-relief and Color Shaded-relief maps of the Willamette Valley, Oregon
}

\section{(digital database description)}

By

R. W. Givler and R. E. Wells

Open - File Report 01-294

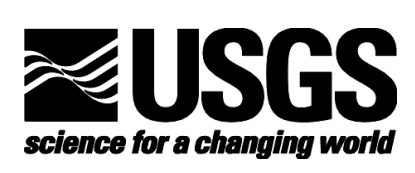

${ }^{1}$ U.S. Geological Survey, 345 Middlefield Rd. MS-975, Menlo Park, CA 94025 


\title{
U.S. Department of the Interior
}

\author{
Gale A. Norton, Secretary
}

\section{U.S. Geological Survey}

Charles Groat, Director

This report is preliminary and has not been reviewed for conformity with U.S. Geological Survey editorial standards or with the North American Stratigraphic Code. Any use of trade, product, or firm names is for descriptive purposes only and does not imply endorsement by the U.S. Government.

This database, identified as "Shaded-relief and Color shaded-relief maps of the Willamette Valley, Oregon" has been approved for release and publication by the Director of the USGS. Although this database has been subjected to rigorous review and is substantially complete, the USGS reserves the right to revise the data pursuant to further analysis and review.

Furthermore, it is released on condition that neither the USGS nor the United States Government may be held liable for any damages resulting from its authorized or unauthorized use.

To obtain this pamphlet, contact:

USGS Information Services

Box 25286

Denver Federal Center

Denver, CO 80225

303-202-4700

303-202-4693 FAX

U.S. Department of the Interior

U.S. Geological Survey 


\section{Introduction}

This Open-File report is released as a digital map database. This pamphlet serves to introduce and describe the digital data. The report does include, however, PostScript plot files which contain images of the map sheets. These images also contain a brief explanation describing the geology and physiography of the area. For those only interested in a paper plot of information contained in the database or in obtaining the PostScript plot files, please see the section entitled "For Those Who Don't Use Digital Map Databases" below.

This digital map database is a compilation of newly published 10-meter digital elevation model data (DEM) for western Oregon and represents the physiography of the Willamette Valley. The text containing a short description of the geology and physiography is provided on the map sheets. Open File Report 01-294 may be found on the web at:

http://geopubs.wr.usgs.gov/open-file/of01-294/

The scale of the source maps limits the spatial resolution (scale) of the database to 1:24,000 or smaller. The content and character of the database and 10-meter DEM data, as well as three methods of obtaining the data, are described below.

\section{For those who don't use digital geologic map databases}

For those interested in shaded-relief maps of the Willamette Valley who do not use an ARC/INFO compatible Geographic Information System (GIS), we have provided two sets of plot files containing images of much of the information in the database. There is a set of images in PostScript format and another in Adobe Acrobat PDF format (see the sections "PostScript plot files" and "PDF plot files" below).

Those interested, who have computer capability, can access the plot file packages in any of the three ways described below (see the section "Obtaining the digital database and plot file packages"). However, it should be noted the plot file packages do require gzip and tar utilities to access the plot files. Therefore additional software, available free on the Internet, may be required to use the plot files (see section "Tar files").

Those without computer capability can obtain plots of the map files through USGS plot-on-demand service for digital maps (see section "Obtaining plots from USGS Open-File Services") or from an outside vendor (see section "Obtaining plots from an outside vendor").

\section{Digital Open-File contents}

This Open-File report consists of three types of digital packages: 1) PostScript Plotfile Packages, 2) PDF Plotfile Packages, and 3) Digital Database - Metadata Packages. We are publishing these maps at two different scales because the map sheets at 1:125,000 scale are 36" wide and 74" long. We realize many users might not need a map of these dimensions. Although we recommend plotting the larger map, we have created a map half the length at $1: 250,000$ scale (36" $\mathrm{x} 40$ "). Maps plotted at the smaller scale differ only in page size and labeling of the scale bars. Further, the maps plotted at 1:250,000 scale are actually created at 1:125,000 in ARC/INFO's arcplot module and reduced to the lower scale by using the scale option with the postscript command. Thus the Adobe Illustrator files containing the map collar information all have the 1:125,000 map scale dimensions (74" x 36"). Below is a detailed description of the Plotfile Packages release with this publication. 


\section{PostScript plot file packages}

Package 1- (of01294ps1.tar.gz)

This package contains the image for the color shaded-relief map (Sheet 1) at 1:125,000 scale in PostScript and the readme in PDF format (see below for more information on PostScript plot files):

wvc125.ps A PostScript plottable file containing an image of the Willamette Valley color shaded-relief map (Sheet 1) at a scale of 1:125,000 (36"x74").

readme.pdf A PDF file of this document.

Package 2 - (of01294ps2.tar.gz)

This package contains the image for the gray-scale shaded-relief map (Sheet 2) at 1:125,000 scale in PostScript and the readme in PDF format (see below for more information on PostScript plot files):

wvs125.ps A PostScript plottable file containing an image of the Willamette Valley gray-scale shaded-relief map (Sheet 2) at a scale of 1:125,000 (36"x74").

readme.pdf A PDF file of this document.

Package 3 - (of01294ps3.tar.gz)

This package contains the image for the color shaded-relief map (Sheet 1) at 1:250,000 scale in PostScript and the readme in PDF format (see below for more information on PostScript plot files):

wvc250.ps A PostScript plottable file containing an image of the Willamette Valley color shaded-relief map (Sheet 1 ) at a scale of 1:250,000 (36"x40").

readme.pdf A PDF file of this document.

Package 4 - (of01294ps4.tar.gz)

This package contains the image for the gray-scale shaded-relief map (Sheet 2) at 1:250,000 scale in PostScript and the readme in PDF format (see below for more information on PostScript plot files):

wvs250.ps A PostScript plottable file containing an image of the Willamette Valley gray-scale shaded-relief map (Sheet 2) at a scale of 1:250,000 (36"x40").

readme.pdf $\quad$ A PDF file of this document.

\section{PDF plotfile packages}

Package 1: (of01294pdf1.tar.gz)

This package contains the images described here in PDF format (see below for more information on PDF plot files):

wvc125.pdf

wvs125.pdf

readme.pdf
A PDF containing an image of the color shaded-relief map at a scale of 1:125,000 (Sheet 1).

A PDF containing an image of the gray-scale shaded-relief map at a scale of 1:125,000 (Sheet 2).

A PDF of this document. 
Package 2: (of01294ps2.tar.gz)

This package contains the images described here in PDF format (see below for more information on PDF plot files):

wvc250.pdf

wvs250.pdf

readme.pdf
A PDF containing an image of the color shaded-relief map at a scale of 1:250,000 (Sheet $3)$.

A PDF containing an image of the gray-scale shaded-relief map at a scale of 1:250,000 (Sheet 4).

A PDF of this document.

\section{Digital database - metadata packages}

The database packages include the database files and the compiled 10-meter DEM data for the Willamette Valley maps. The digital maps, or coverages, along with their associated INFO directory have been converted to uncompressed ARC/INFO export files. ARC export files promote ease of data handling, and are usable by some Geographic Information Systems in addition to ARC/INFO (see below for a discussion of working with export files). Raster data are stored in ARC grid format rather than export format to reduce file size. The grids were further converted into decimeter integer grids to reduce size. If you are interested in learning more about the compilation of the 10-meter DEM data please see the section titled 'Data Compilation'. The ARC export files and associated ARC/INFO coverages, grids, and directories, as well as the additional digital material included in the database are described below:

Digital Database Package 1: (of01294db1.tar.gz)

\begin{tabular}{lc}
$\begin{array}{l}\text { ARC/INFO } \\
\text { export file }\end{array}$ & $\begin{array}{c}\text { Resultant } \\
\text { Coverage }\end{array}$ \\
\hline will_clip.e00 & will_clip \\
will_clip1.e00 & will_clip1
\end{tabular}

\section{ARC/INFO grids}

wil_hue

wil_sat

wil_val

wil_hil

\section{Description of Coverage}

Boundary coverage for the Willamette Valley maps

Boundary coverage for the Willamette Valley maps with areas of nodata. This coverage is used in the grid routine that excludes areas outside the irregular map boundary (see Data Compilation section below)

\section{Description of Grid}

Grid containing the hue information for the color shadedrelief gridcomposite (cellsize 20-meters)

Grid containing the saturation information for the color shaded-relief gridcomposite (cellsize 20-meters)

Grid containing the value information. This grid corresponds to the hillshading information in the color shaded-relief gridcomposite (cellsize 20 -meters)

A grid containing the hillshade information used to produce Sheet 1 (cellsize 20-meters) 
This database package also includes the following files:

ASCII text files, including explanatory text, PostScript plot files, Encapsulated PostScript files from Adobe Illustrator 8 used as map collars and Figures (EPS) for the report, ARC Macro Language (AML) files for plotting maps, custom ARC shadesets, and text docuements describing the data (metadata.txt and readme.txt) (Note: The metagraphics files (.gra) are not present in this data package):

metadata.txt The metadata

metadata.pdf The metadata as a PDF file

readme.txt This file

readme.pdf This file as a PDF file

will_exp.doc Microsoft word version of the text on the map sheets, including references.

wv1a.eps $\quad$ Encapsulated PostScript Adobe Illustrator 8 file (map collar) for the Willamette Valley color shaded-relief map plotted at 1:125,000 scale. Contains figures, text, and a brief explanation (Sheet 1)

wv2a.eps Encapsulated PostScript Adobe Illustrator 8 file (map collar) for the Willamette Valley grayscale shaded-relief map plotted at 1:125,000 scale. Contains figures, text, and a brief explanation (Sheet 2)

wvlb.eps

Encapsulated PostScript Adobe Illustrator 8 file (map collar) for the Willamette Valley color shaded-relief map plotted at 1:250,000 scale. Contains figures, text, and a brief explanation (Sheet 3)

wv2b.eps Encapsulated PostScript Adobe Illustrator 8 file (map collar) for the Willamette Valley grayscale shaded-relief map plotted at 1:250,000 scale. Contains figures, text, and a brief explanation (Sheet 4)

will_plot.aml Plot AML generates uncompressed PostScript of Willamette Valley shaded-relief maps at 200 dpi. This aml asks the user to specifiy the scale and either Sheet 1,2,3, or 4.

mapefix_aml.aml Aml called by will_plot.aml, which sucks in the mapextent by $10 \%$. Anything outside the map boundary is not drawn using the clipmapextent command. By default, ARC adds $10 \%$ extra to the map boundary. This short AML eliminates that extra 10\% (written by R. Haugerud)

geo.prj

Projection file that defines a geographic projection

utm.prj

Projection file that defines a Universal Transmercator (UTM) projection

geo2utm.prj

A projection file that projects files from geographic to UTM

utm2geo.prj

A projection file that projects files from UTM to geographic

hue_table

A remap table used to assign elevation ranges a hue value (explained in Data compilation section below)

sat_table

A remap table used to assign elevation ranges a saturation value (explained in Data compilation section below)

uncom

Parameter file in will_plot.aml to uncompress PostScript file

info

Arc/Info directory 
$\log$

hsv_shade85.shd

hsv_shade90.shd

hsv85u.ps

hsv90u.ps
Arc/Info log file

Arc/Info shadeset (custom) based on the HSV Tektronix color model with a set value of 85 . (See text for reasons why the value is set constant for this and the below shadeset)

Arc/Info shadeset (custom) based on the HSV Tektronix color model with a set value of 90

PostScript images of the shadeset hsv_shade85.shd created in ARC/INFO

PostScript images of the shadeset hsv_shade90.shd created in ARC/INFO

Digital Database Package 2: (of01294db2.tar.gz)

ARC/INFO grids

wil_grid

info

\section{Description of Grid}

Grid containing the original 10-meter DEM data compilation (decimeter grid with a cellsize of 10-meters)

Arc/Info directory

\section{Tar files}

The eight data packages described above are stored in tar (UNIX tape archive) files. A tar utility is required to extract the database from the tar file. This utility is included in most UNIX systems, and can be obtained free of charge over the Internet from Internet Literacy's Common Internet File Formats Webpage (http://www.matisse.net/files/formats.htmI). The tar files have been compressed, and may be uncompressed with gzip, which is available free of charge over the Internet via links from the USGS Public Domain Software page (http://edcwww.cr.usgs.gov/doc/edchome/ndcdb/public.html). If you are using a PC, a software package called cygwin will provide a 'UNIX like' environment on a PC. By using cygwin these files may be untarred and uncompressed. Download this software for free at http://sources.redhat.com/cygwin/ Additionally, a free software program for a PC with a graphical user interface called winzip will do many of the same functions as the above programs. Download winzip at http://www.winzip.com/. When the tar file is uncompressed and the data is extracted from the tar file, a directory is produced that contains the data in the package as described above. The specific names of the tarred and gziped files are listed below:

Name of
compressed
tar file

of01294ps1.tar.gz of01294ps2.tar.gz of01294ps3.tar.gz of01294ps4.tar.gz of01294pdf1.tar.ga of01294pdf2.tar.gz of01294db1.tar.gz of01294db2.tar.gz

Size of
compressed
tar file
(uncompressed)

$57.3 \mathrm{MB}(299 \mathrm{MB})$
$34 \mathrm{MB}(100 \mathrm{MB})$
$52 \mathrm{MB}(267 \mathrm{MB})$
$30.7 \mathrm{MB}(89.7 \mathrm{MB})$
$37.2 \mathrm{MB}(38.1 \mathrm{MB})$
$34.4 \mathrm{MB}(35.2 \mathrm{MB})$
$121 \mathrm{MB}(210 \mathrm{MB})$
$166 \mathrm{MB}(256 \mathrm{MB})$

\section{Directory \\ produced when extracted from tar file}

ofr_ps 1/

ofr_ps $2 /$

ofr_ps3/

ofr_ps4/

ofr_pdf1/

ofr_pdf2/

ofr $\mathrm{db} 1 /$

ofr_db2/

\section{Data package contained}

PostScript Plotfile Package 1

PostScript Plotfile Package 2

PostScript Plotfile Package 3

PostScript Plotfile Package 4

Portable Document Format Package 1

Portable Document Format Package 2

Digital Database Package \& Metadata 1

Digital Database Package \& Metadata 2 


\section{PostScript plot files}

For those interested in the shaded-relief maps of the Willamette Valley who don't use an ARC/INFO compatible GIS system we have included four separate data packages with a PostScript plot file and this document in each. Because this release is primarily a digital database, the plot file (and plots derived there from) has not been edited to conform to U.S. Geological Survey standards. The PostScript images in PostScript Package 1 or $\mathbf{2}$ contain sheets 1 or 2 measuring 74 by 36 inches, so these files require a large plotter to produce paper copies at the intended scale $(1: 125,000)$. Further, the PostScript images in PostScript Packages 3 and $\mathbf{4}$ contain sheets 3 or 4 measuring 36 by 40 inches, so these files also require large plotters at the intended scale. When downloading and working with these files please be aware of their page and file size. If you intend to download these files and send them to a plotter, please be sure the plotter is equipped to handle the above page size and memory requirements.

The PostScript plot files for maps were produced by the PostScript command using the uncompressed option in ARC/INFO version 8.0.2 for UNIX. The map collars (wv1a.eps, wv2a.eps, wv1b.eps, and wv2b.eps) contain a color plot of the figures, scale, and text, but no map image as Encapsulated PostScript Files.

The final ARC/INFO PostScript files (wvc125.ps, wvs125.ps, wvc250.ps, and wvs250.ps) contain Encapsulated PostScript files generated by Adobe Illustrator version 8 (above) and placed into the ARC/INFO graphic metafile by an ARC plotting AML.

The Plot AML (will_plot.aml) uses the coverages and grids to produce a printable PostScript file of this Open File report. The Plot AML will ask the user to specify what scale and which map sheet should be plotted. The mape_fixaml.aml sucks in the map extent by $10 \%$ so the clipmapextent command actually clips at the map boundary. By default the map extent is defined by the extent of the map plus $10 \%$. Called by the Plot AML, this AML eliminates the excess space outside the map boundary defined by the map extent. Ralph Haugerud wrote the mapefix_aml.aml originally.

\section{PDF plot files}

We have included a second set of digital packages containing PDF versions of the PostScript map sheets described above. Adobe Acrobat PDF (Portable Document Format) files are similar to PostScript plot files in that they contain all the information needed to produce a paper copy of a map and they are platform independent. Their principal advantage is that they require less memory to store and are therefore quicker to download from the Internet. In addition, PDF files allow for printing of portions of a map image on a printer smaller than that required to print the entire map without the purchase of expensive additional software. All PDF files in this report have been created from PostScript plot files using Adobe Acrobat Distiller. In test plots, we have found that paper maps created with PDF files contain almost all the detail of maps created with PostScript plot files. We would, however, recommend that those users with the capability to print the large PostScript plot files use them in preference to the PDF files.

To use PDF files, the user must get and install a copy of Adobe Acrobat Reader. This software is available free from the Adobe website (http://www.adobe.com). Please follow the instructions given at the website to download and install this software. Once installed, the Acrobat Reader software contains an on-line manual and tutorial.

There are two ways to use Acrobat Reader in conjunction with the Internet. One is to use the PDF reader plug-in with your Internet browser. This allows for interactive viewing of PDF file images within your browser. This is a very handy way to quickly look at PDF files without downloading them to your hard disk. The second way is to download the PDF file to your local hard disk, and then view the file with Acrobat Reader. **We strongly recommend that large map images be handled by downloading to your hard disk, because viewing them within an Internet browser tends to be very slow.**

To print a smaller portion of a PDF map image using Acrobat Reader, it is necessary to cut out the portion desired using Acrobat Reader and the standard cut and paste tools for your platform, and then to paste the portion of the image into a file generated by another software program that can handle images. Most word processors (such as Microsoft Word) will suffice. The new file can then be printed. Image conversion in the cut and paste process, as well as changes in the scale of the map image, may result in loss of image quality. However, test plots have proven 
adequate. Another approach, would be to use Adobe Acrobat reader to clip images into the desired dimensions and then print to your printer directly from this software.

\section{Digital database format}

The databases in this report were compiled in ARC/INFO, a commercial Geographic Information System (Environmental Systems Research Institute, Redlands, California), with version 3.0 of the menu interface ALACARTE (Fitzgibbon and Wentworth, 1991, Fitzgibbon, 1991, Wentworth and Fitzgibbon, 1991). The files are in either GRID (ARC/INFO raster data) format or COVERAGE (ARC/INFO vector data) format. Coverages are stored in uncompressed ARC export format (ARC/INFO version 8.0.1 for Unix). ARC/INFO export files (files with the .e00 extension) can be converted into ARC/INFO coverages in ARC/INFO (see below) and can be read by some other Geographic Information Systems, such as MapInfo via ArcLink and ESRI's ArcView (version 1.0 for Windows 3.1 to 3.11 is available for free from ESRI's web site: http://www.esri.com). The digital compilation was done in version 8.0.2 of ARC/INFO for UNIX.

\section{Obtaining the Digital Database and Plotfile Packages}

The digital data can be obtained in any of three ways:

a. From the Western Region Geologic Information Web Page

b. Anonymous ftp over the Internet

c. Request a CD

To obtain tar files of database or plot file packages from the USGS web pages:

The U.S. Geological Survey now supports a set of graphical pages on the World Wide Web. Digital publications (including this one) can be accessed via these pages. The location of the main Web page for the entire USGS is

http://www.usgs.gov

The Web server for digital publications from the Western Region is

http://geopubs.wr.usgs.gov

Go to

http://geopubs.wr.usgs.gov/open-file/of01-294

to access this publication. Besides providing easy access to the entire digital database, the Western Region Web page also affords easy access to the PostScript plot files for those who do not use digital databases (see below).

\section{To obtain tar files of database or plot file packages by ftp:}

The files in these reports are stored on the U.S. Geological Survey Western Region FTP server. The Internet ftp address of this server is:

\section{ftp://geopubs.wr.usgs.gov}

The user should log in with the user name anonymous and then input their e-mail address as the password. This will give the user access to all the publications available via ftp from this server.

The files in this report are stored in the subdirectory:

pub/open-file/of01-294 


\section{To obtain tar files of database or plot file packages on CD:}

Database files, PostScript plot files, and related files can be obtained

by sending a request for a compact disk (CD) and including a return address to:

Willamette Valley Shaded-relief Plotfiles

Pacific Northwest Geologic Mapping Project

c/o Database Coordinator

U.S. Geological Survey

345 Middlefield Road, M/S 975

Menlo Park, CA 94025

\section{Do not omit any part of this address!}

NOTE: Be sure to include with your request the exact names, as listed above, of the tar files you require. An OpenFile Report number is not sufficient, unless you are requesting the database package, plot file package for the report, and PDF package. This is especially important because this open-file report has several plotfile packages.

The compressed tar files will be returned on the compact disk(s).

\section{Obtaining plots from a commercial vendor}

Those interested in the shaded-relief maps of the Willamette Valley but who use neither a computer nor the Internet, can still obtain the information. We will provide the PostScript plot files on digital compact disk (details below) for use by commercial vendors who can make large-format plots. Send request for a disk (CD) with return address to:

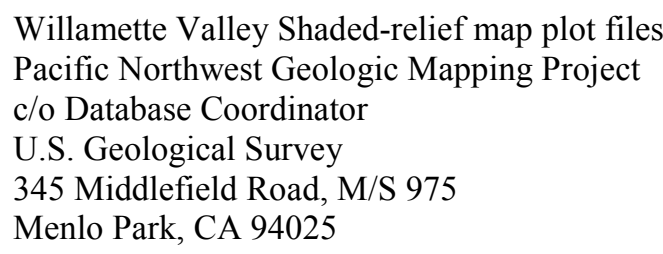

\section{Do not omit any part of this address!}

The compressed tar files will be returned on the compact disk(s).

Make sure your vendor is capable of reading compact disks and PostScript plot files. Important information regarding compact disk format is included in the sections "Database Release Format", "Tar Files", and "PostScript Plot Files" above, so be certain to provide a copy of this document to your vendor.

\section{Obtaining plots from USGS Open-File Services}

U.S. Geological Survey is providing a plot-on-demand service for map files, such as those described in this report, through Open-File Services. In order to obtain plots, contact Open-File Services at:

\section{USGS Information Services \\ Box 25286 \\ Denver Federal Center \\ Denver, CO 80225-0046}

(303) 202-4200

1-800-USA-MAPS

FAX: (303) 202-4695

e-mail: infoservices@usgs.gov 
Be sure to include with your request the Open-File Report number and the exact names, as listed in the Database Contents section above, of the plot files you require. An Open-File Report number and its letter alone may not be sufficient, unless you are requesting plots of all the plot files for that report. The prices for plot-on-demand services are listed at (http://rockyweb.cr.usgs.gov/mod/order.html).

\section{Converting ARC export files}

ARC export files are converted to ARC coverages by using the ARC command IMPORT with the option COVER. ARC export files can also be read by some other Geographic Information Systems. Please consult your GIS documentation to see if you can use ARC export files and the procedure to import them.

\section{Digital compilation}

Digital Elevation Model Data: The 10-meter DEM data for western Oregon was obtained from the EROS Data Center in Sioux Falls South Dakota. The DEM data for the Willamette Valley is released to the public with this Open-File Report http://geopubs.wr.usgs.gov/open-file/of01-294. If you are interested in just the DEM data please download the compiled 10-meter DEM data package (of01-294db1.tar.gz). The .dem files were compiled in ARC/INFO's grid module. The z-values were converted to meters from feet, if necessary, and subsequently converted to decimeter integer grids. This procedure decreases the data size significantly. These data were then clipped in grid to conform to the present map boundary. Additional 10-meter DEM data for Washington was added to the Oregon data. Washington data was downloaded from http://duff.geology.washington.edu/data/index.html, For plotting purposes the data was down-sampled to a cellsize of 20-meters. A noticeable difference in data quality was not seen in plots at either 1:125,000 or 1:250,000 scale using the larger cellsize. Note, if you download the gridded data it will have a 10-meter cellsize.

Creating color shaded-relief maps: All the shaded-relief maps were created in ARC/INFO's Grid module using the hillshade, slice, con, and gridcomposite commands. Using the Tektronix HSV color model, the appearance of a hillshade with geology or in this case physiography is made possible. The HSV model consists of three different grids: 1) Hue, 2) Saturation, and 3) Value. Hue specifies the color on a 360 degree color wheel. Saturation is the amount of the color, defined by Hue, to be plotted (1-100). No saturation is white regardless of color. Value is the amount of black (0-100) present in that color. Zero value is black and 100 is no black at all. Using this color model, three grids can be made that represent one of these three parameters. They can then be combined to make one color. This is done through the gridcomposite command with the hsv option. The hue and saturation grids were generated using the below slice command with the tables option (everything in " marks a variable the user must specify and omit the " from actual grid syntax):

Grid: 'hue_grid' = slice('wil_grid', table, 'hue_table')

The 'hue_grid' is the grid generated from the above command. This intermediate file is not present in this data set. The 'wil_grid' is the 10-meter grid of the compiled DEM data. The 'hue_table' is a remap table that assigns elevation ranges to user specified hue values. The above command will generate a separate grid with values specified by the 'hue_table'. For example, the beginning of the 'hue_table' looks like this:

$0: 150$

$50: 140$

$100: 135$

$150: 135$

$200: 125$

$250: 120$

.

$12000: 30$

$132000: 30$

(end of table) 
Using the above remap table any values less than 0 will be assigned a hue value of 150 and any value between 200250 will be assigned to hue value of 120 . The saturation grid is determined the same way, except the saturation remap table ('sat_table') is substituted for the 'hue table'. Both the remap tables hue table and sat table are provided in the database and metadata package. Using this method the user has full control over elevation ranges and color distribution for a color shaded-relief map. Further, by studying the physiography of a given area one can pick custom elevation ranges and colors which highlight areas of importance. When beginning this process it is important to study the HSV model to determine how it changes from one color to another. This will allow for subtle color variations rather than hard color contrasts. In the metadata package, there are both the PostScript and the shadeset files for two HSV shadesets. More information about the HSV color model and the usage for the below commands can be found with Open-file report 98-487 (http://pubs.usgs.gov/openfile/of98-487/haug2.html) or in the ARC/INFO help. Saturation values less than 70-80, we believe, produced the best looking pastel colored maps.

Integrating the hillshaded topography into a color shaded-relief map is done through the value grid. Remember the value grid is amount of black in each color. A hillshade grid can be sliced into values of zero to 100 and can be used to calculate the amount of black independent of the other grids. To create a value grid from a DEM, use the following sequence of commands (everything in " marks a variable the user must specify and omit the " from actual grid syntax):

Grid: 'hillshade' = hillshade ('wil_grid', 300, 60, SHADE, 0.3)

Grid: 'temp1' = slice('hillshade', eqinterval, 100)

Grid: 'value-grid' = con(isnull('temp1'),100,'temp1')

The 'hillshade' grid is the intermediate output grid and the input 'wil_grid' is the DEM grid in decimeters. The 300 is the azimuth of your illumination source and the 60 is the illumination angle above the horizon. The 0.3 is the $\mathrm{z}$ factor that sets the amount of vertical exaggeration. With color shaded-relief maps a higher illumination angle value (around 60) produces a grid with lighter more vibrant colors. For this publication, three times vertical exaggeration was selected, to emphasize flood and fluvial features, by setting the $\mathrm{z}$-factor parameters at 0.3 (remember that these are decimeter grids so a vertical exaggeration needs to be in tenths). The hillshade command pushes out a grid with 255 gray values. The second line of code pushes those values into 100 equally spaced values. The third line is a con statement that turns any nodata, in the temp 1 grid, into a value of 100 or white.

Stream coverages downloaded from the Internet (www.streamnet.org) were added to the hue and saturation grids using a series of conditional statements or con statements. It should be noted, a subset of the original stream coverages is presented with this report as a part of the composite grids. First, both polygon and line coverages representing the important rivers in the Willamette valley were made into a grids using the LINEGRID and POLYGRID commands. These grids were then combined into one grid with the rivers representing one cellvalue of 9, the areas inside the map boundary had a cellvalue of 1, and the areas outside the map boundary were 999 . Lastly, the following lines of code were used to merge the hue and saturation grids with the river grids:

Grid: 'hue1' $=$ con('riv-grid' $==9,200$,'riv-grid' $==1$,'hue_grid','riv_grid' $==999,100$,'hue-grid')

Grid: 'sat1' $=\operatorname{con}$ ('riv-grid' $==9,75$,'riv-grid' $==1$,'sat_grid','riv_grid' $==999,0$,'sat-grid')

The first set of code in the con statement ('riv-grid' $==9,200$ ) is setting any value equal to nine in the 'riv-grid' to 200 in the 'huel' grid. This converts the rivers to a hue value of 200. The next peace of the statement ('riv-grid' == 1,'hue_grid') specifies that any value equal to one in the 'riv-grid' should be replaced with the 'hue_grid' (see above commands) in the output 'huel'. This sets the areas within the map boundary that are not rivers to the 'hue_grid' values. Next statement ('riv_grid' $==999,100$ ) sets the values equal to 999 in the 'riv-grid' to 100 in the output 'hue1' grid. Values in 'riv_grid' equal to 999 represent the areas outside the map boundary. The last portion of this statement sets everything that wasn't set by the previous three statements to values in the 'hue_grid'. The line of code for the saturation grid (sat1) does essential the same thing except the saturation values are 75 not 200 . The most import line of this code for the saturation gird is the third statement ('riv-grid' $==999,0$ ). This statement sets the areas outside the map boundary to a saturation of zero, which makes these areas white. A gridcomposite with 
these new grids will plot the color shaded-relief with the streams 'underneath' the hillshading. We took this approach because previous plots with the river polygons and lines on top of the shaded relief looked artificial. Please note, the 'riv_grid' is not released separately with this dataset. If you are interested in compiling a river dataset for the Willamette Valley shapefiles are available at 1:100,000 scale at www.streamnet.org

\section{Creating gray-scale shaded-relief maps:}

The gray-scale shaded-relief maps were created in ARC/INFO's grid module with the hillshade command. The below command was used to generate these maps (everything in " marks a variable the user must specify and omit the " from actual grid syntax):

$$
\text { Grid: 'wil_hil' = hillshade ('wil_grid', 300, 45, SHADE, 0.3) }
$$

The 'wil_hil' grid is the output grid and the input 'wil_grid' is the DEM grid in decimeters. Both grids are released with this report (ofr_db1.tar.gz and ofr_db1.tar.gz). The 300 is the azimuth of your illumination source and the 45 is the illumination angle above the horizon. The illumination angle was changed from 60 to 45 for the gray-scale plots because the lower value produced darker images, which the authors felt was more desirable for gray-scale plots. The 0.3 is the $\mathrm{z}$-factor that sets the amount of vertical exaggeration. The wil_plot.aml draws this image using the gridpaint command and the below syntax (everything in " marks a variable the user must specify and omit the " from actual grid syntax):

Arcplot: gridnodatasymbol white

Arcplot: gridpaint wil_hil \# \# \# gray

The gridnodatasymbol comand specifies to Arcplot those areas of nodata, or places with no shaded-relief, will be plotted white. If you are interested in what context this command was used please study where in the will_plot.aml the gridpaint command was used (line 149).

Plotting: The plot aml runs in ARC/INFO and calls the coverages, grids, and EPS files to make an uncompressed PostScript file. If you are interested in the syntax that produced the cartographic elements presented on these maps, please refer to the will_plot.aml or the help in ARC/INFO. A digital layout or map collar was made with Adobe Illustrator. All maps are in Universal Transmercator, zone 10, units meters, and plotted at either 1:125,000 or 1:250,000 scale. The pamphlet that describes the physiography and general geology was a Microsoft word document before it was added to the Adobe Illustrator file.

\section{Spatial resolution}

Uses of these digital data should not violate the spatial resolution of the original data. These digital elevation models were created from 7.5" topographic quadrangles. Although the form of the data removes the constraint imposed by the scale of a paper map, the detail and accuracy inherent in map scale are also present in the digital data. The fact that this database was edited at a scale of 1:24,000 means that higher resolution information is not present in the dataset. Plotting at scales larger than 1:24,000 will not yield greater real detail, although it may reveal fine-scale irregularities below the intended resolution of the database. Similarly, where this database is used in combination with other data of higher resolution, the resolution of the combined output will be limited by the lower resolution of these data. Further, it should be noted the grids used to generate the color shaded-relief image have a grid cell size of 20 meters, not 10 meters. If you are interested in using this data at 1:24,000 scale be sure to download from this publication the 10-meter grids and create your own color or gray-scale maps.

\section{Database specifics}

The map databases consist of ARC coverages and supporting INFO files, which are stored in a Universal Transmercator projection (UTM) (Table 1). Digital tics define a grid of latitude and longitude in the will_clip coverage corresponding with map boundaries.

Table 1 - Map Projection 
The maps are stored in Universal Transmercator projection (UTM)

$\begin{array}{ll}\text { PROJECTION UTM } & \\ \text { UNITS METERS } & \text {-on the ground } \\ \text { ZONE 10 } 10 & \text {-UTM zone } \\ \text { DATUM } & \text {-NAD27 } \\ \text { SPHEROID } & \text {-CLARKE1866 } \\ \text { PARAMETERS } & \\ \text { END } & \end{array}$

The content of the database can be described in terms of the lines and the areas that compose the map. Descriptions of the database fields use the terms explained in Table 2.

Table 2 - Field Definition Terms

ITEM NAME name of the database field (item)

WIDTH maximum number of digits or characters stored

OUTPUT output width

TYPE B-binary integer, F-binary floating point number,

N. DEC. number of decimal places maintained for floating point numbers

\section{Lines}

The lines (arcs) are recorded as strings of vectors and are described in the arc attribute table (Table 3). They define the boundaries of the map.

Table 3 - Content of the Arc Attribute Tables

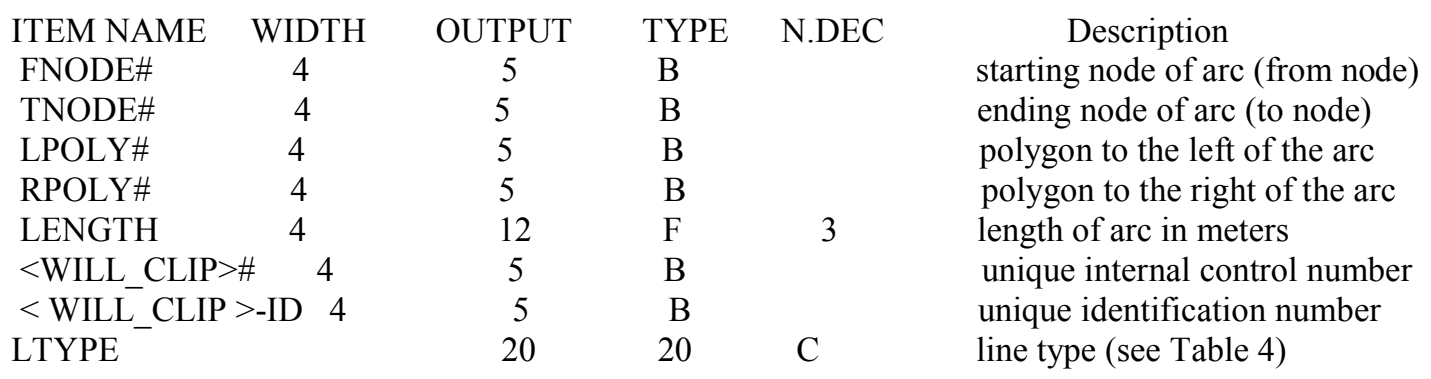

Table 4 - Line Types Recorded in the LTYPE Field.

will_clip

map boundary

will_clip1

map boundary

outside map boundary

\section{Areas}

Map areas (polygons) are described in the polygon attribute table (Table 5). Note that ARC/INFO coverages cannot 
contain both point and polygon information, so only coverages with polygon information will have a polygon attribute table, and these coverages will not have a point attribute table.

Table 5 - Content of the Polygon Attribute Tables

\begin{tabular}{|c|c|c|c|c|c|}
\hline ITEM NAME & NIDTH & OUTPUT & TYPE & N.DEC & Description \\
\hline AREA & 4 & 12 & $\mathrm{~F}$ & 3 & area of polygon in square meters \\
\hline PERIMETER & 4 & 12 & $\mathrm{~F}$ & 3 & length of perimeter in meters \\
\hline$<$ WILL_CLIP $>\#$ & 4 & 5 & B & & unique internal control number \\
\hline$<$ WILL_CLIP $>$-ID & 4 & 5 & B & & unique identification number \\
\hline TYPE & 35 & 35 & $\mathrm{C}$ & & unit label \\
\hline
\end{tabular}

\section{Will_clip.pat - type}

yes - indicates areas inside the map boundary.

no - indicates areas out side the map boundary.

(no other types were specified)

\section{Will_clip1.pat - type}

yes - indicates areas inside the map boundary.

no - indicates areas outside the map boundary.

(no other types were specified)

\section{Acknowledgments}

We wish to thank Karen Wheeler for the digital review of this Open File report.

\section{References Cited}

Fitzgibbon, T.T., 1991, ALACARTE installation and system manual (version 1.0): U.S. Geological Survey OpenFile Report 91-587B.

Fitzgibbon, T.T., and Wentworth, C.M., 1991, ALACARTE user interface - AML code and demonstration maps (version 1.0): U.S. Geological Survey Open-File Report 91-587A.

Haugerud, R., and Greenberg, H.M., 1998, Recipes for Digital Cartography: Cooking with DEMs: U.S. Geological Survey Open-File Report 98-487, U.S. Geological Survey, available at http://pubs.usgs.gov/openfile/of98-487/haug2.html

Wentworth, C.M., and Fitzgibbon, T.T., 1991, ALACARTE user manual (version 1.0): U.S. Geological Survey Open-File Report 91-587C. 